\title{
The ZEPLIN III Detector; Results from Surface Calibrations
}

T. J. Sumner ${ }^{\mathrm{a}}$, D. Yu. Akimov ${ }^{\mathrm{b}}$, G. J. Alner ${ }^{\mathrm{c}}$, H. M. Araújo, A. Bewick ${ }^{\mathrm{a}}$, C. Bungau, A. A. Burenkov ${ }^{\mathrm{b}}$, M. J. Carson ${ }^{\mathrm{d}}$, H. Chagani ${ }^{\mathrm{d}}$, V. Chepel ${ }^{\mathrm{e}}$, D. Cline ${ }^{\mathrm{f}}$, D. Davidge ${ }^{\mathrm{a}}$, E. Daw ${ }^{\mathrm{d}}$, J. Dawson ${ }^{\mathrm{a}}$, T. Durkin ${ }^{\mathrm{c}}$ B. Edwards, T. Gamble ${ }^{d}$, C. Chagg, R. J. Hollingworth ${ }^{\mathrm{d}}$, A. S. Howard ${ }^{\mathrm{a}}$, W. G. Jones ${ }^{\mathrm{a}}$, M. Joshi ${ }^{\mathrm{a}}$, K. Mavrokoridis $^{\mathrm{d}}$, E. Korolkova ${ }^{\mathrm{g}}$, A. Kovalenko ${ }^{\mathrm{b}}$, V. A. Kudryavtsev ${ }^{\mathrm{d}}$, I. S. Kuznetsov ${ }^{\mathrm{b}}$, T. Lawson ${ }^{\mathrm{d}}$, V. N. Lebedenko ${ }^{a}$, J. D. Lewin ${ }^{\mathrm{c}}$ P. Lightfoot ${ }^{\mathrm{d}}$, A. Lindote ${ }^{\mathrm{e}}$, I. Liubarsky ${ }^{\mathrm{a}}$, M. I. Lopes ${ }^{\mathrm{e}}$, R. Lüscher ${ }^{\mathrm{c}}$ J. E. McMillan $^{\mathrm{d}}$, P. Majewski ${ }^{\mathrm{d}}$, B. Morgan ${ }^{\mathrm{d}}$, D. Muna ${ }^{\mathrm{d}}$, A. S. Murphy ${ }^{\mathrm{g}}$, F. Neves ${ }^{\mathrm{e}}$, G. G. Nicklin ${ }^{\mathrm{d}}$, S. M. Paling $^{d}$, J. Pinto da Cunha ${ }^{\mathrm{e}}$, S. J. S. Plank ${ }^{g}$, R. Preece ${ }^{\mathrm{c}}$ J. J. Quenby ${ }^{\mathrm{a}}$, M. Robinson ${ }^{\mathrm{d}}$, C. Silva ${ }^{\mathrm{e}}$, V. N. Solovove, N. J. T. Smith ${ }^{\text {P }}$. F. Smith ${ }^{\mathrm{c}}$ N. J. C. Spooner ${ }^{\mathrm{d}}$, V. Stekhanov ${ }^{\mathrm{b}}$, C. Thorne ${ }^{\mathrm{a}}$, D. R. Tovey ${ }^{\mathrm{d}}$, E. Tziaferi $^{\mathrm{d}}$, R. J. Walker ${ }^{\mathrm{a}}$, H. Wang ${ }^{\mathrm{f}}$, J. White $^{\mathrm{h}}$ and F. Wolfs ${ }^{\mathrm{i}}$

${ }^{a}$ Blackett Laboratory, Imperial College London,UK

${ }^{\mathrm{b}}$ Institute for Theoretical and Experimental Physics, Moscow, Russia

${ }^{c}$ Particle Physics Department, Rutherford Appleton Laboratory, Chilton, UK

${ }^{\mathrm{d}}$ Physics and Astronomy Department, University of Sheffield, UK

${ }^{\mathrm{e}}$ LIP-Coimbra \& Department of Physics of the University of Coimbra, Portugal

${ }^{\mathrm{f}}$ Department of Physics \& Astronomy, University of California, Los Angeles, USA

${ }^{g}$ School of Physics, University of Edinburgh, UK

${ }^{\mathrm{h}}$ Texas A\&M University, USA

${ }^{i}$ University of Rochester, New York, USA

ZEPLIN-III is a direct dark-matter search instrument using liquid xenon as a target. Both the scintillation light and the ionisation charge are measured. The instrument has been built and it is currently being testing in a surface laboratory. We present results from some of the initial testing, including design thermal control system verification, photomultiplier calibration (including spectral measurements with $60 \mathrm{keV}$ photons from ${ }^{241} \mathrm{Am}$ ) and two-phase operation showing simultaneous measurement of scintillation and charge and position reconstruction.

\section{THE ZEPLIN III INSTRUMENT}

ZEPLIN-III is a two-phase (liquid/gas) xenon instrument developed and built by the ZEPLIN Collaboration. ${ }^{1}$ It aims to detect Galactic dark matter in the form of Weakly Interacting Massive Particles, or WIMPs. The instrument is described in $[1,2]$. Once tested at Imperial College,

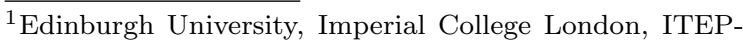
Moscow, LIP-Coimbra, Rochester University, CCLRC Rutherford Appleton Laboratory, Sheffield University, Texas A\&M, UCLA.
}

it will join the ZEPLIN-II [3] and DRIFT-IIa [4] experiments $1100 \mathrm{~m}$ underground in our laboratory at the Boulby mine (North Yorkshire, UK).

Two-phase noble gas emission detectors [5] rely on different particle species generating different relative amounts of vacuum ultra-violet scintillation light and ionisation charge in liquid xenon. WIMPs scatter elastically off Xe atoms, much like neutrons, and a recoiling nucleus produces a different signature to the electron recoil from $\gamma$-rays. ZEPLIN III is a high-field xenon system [6-9]. Monte Carlo simulations $[10,11]$ helped design the 
instrument and to assess its performance [2].

There are four important requirements for a dark matter detector: low energy threshold, particle discrimination, 3-D position reconstruction and low background. Figure $1 \& 2$ show ZEPLINIII at a target concept level and engineering level.
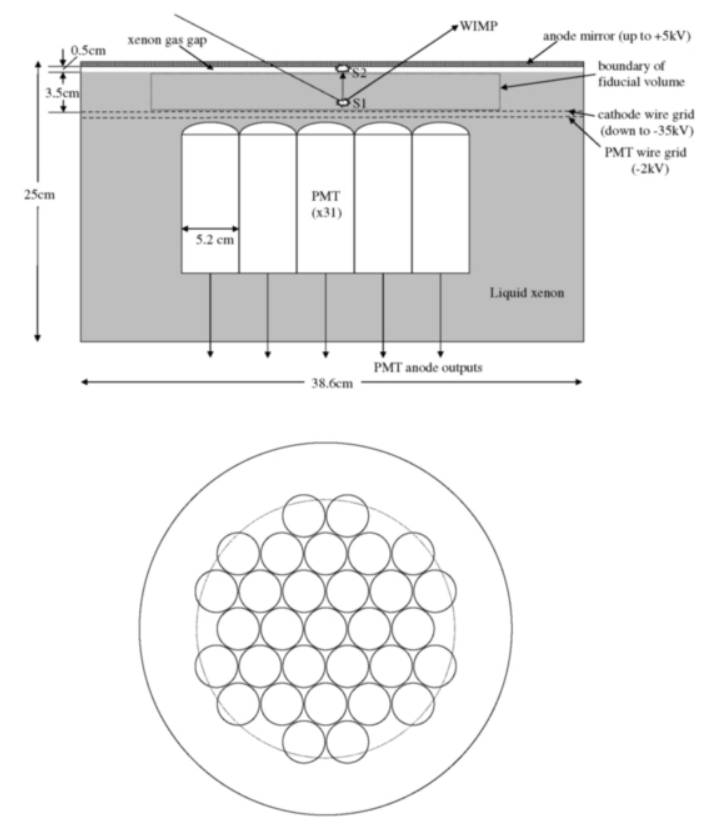

Figure 1. Cross-sections of the target showing the concept. The top panel shows a side view. The bottom panel provides a top view of the PMT arrangement. The fiducial boundary is shown on both.

For low scintillation threshold our photomultipliers (PMTs) are in the liquid, to remove two interfaces with refractive index mismatches, and we use a flat planar geometry to give the highest direct geometrical light collection.

Discrimination comes from measuring scintillation and ionisation for each event. The scintillation output ( $\mathrm{S} 1$ in figure 1) is a prompt, fast signal ( $\tau$ few tens of ns). Escaping ionisation is drifted, by an applied electric field, to the surface and extracted into the gas phase where it causes proportional electroluminescence (S2 in figure 1).
S1 and S2 are separated in time (up to $17 \mu$ s for the $3.5 \mathrm{~cm}$ depth) and are read out by the same PMTs. The ratio S2/S1 depends on the particle species[12] and good 3-D position reconstruction can identify multiple scattering.

The S2 signal pattern in the 312 " diameter PMTs provides sub-cm 2-D $r-r \theta$ resolution even for single electrons extracted from the liquid [18]. The $z$ co-ordinate, to $\sim 50 \mu \mathrm{m}$, comes from the $\mathrm{S} 1$ to $\mathrm{S} 2$ time delay. The 3-D reconstruction defines the fiducial volume without reliance on physical surfaces. The fiducial diameter of $31.2 \mathrm{~cm}$ is shown in figure 1 , and contains $8 \mathrm{~kg}$ of xenon [2].

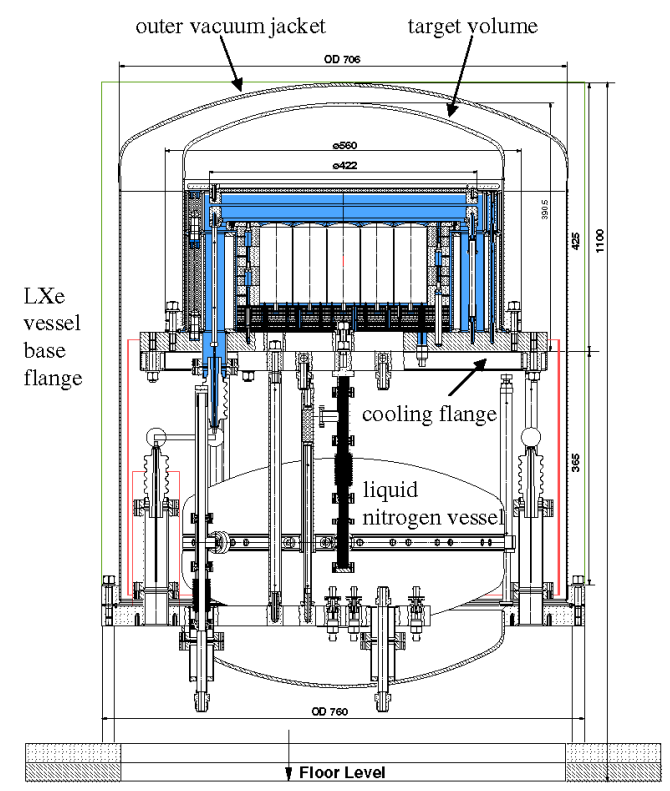

Figure 2. Cross-section of the complete instrument showing the key subsystems. Dimensions are in $\mathrm{mm}$ and ZEPLIN-III is $1100 \mathrm{~mm}$ high with a diameter of $760 \mathrm{~mm}$. Bulk parts are made of C103 OFHC copper.

\section{COMMISSIONING TESTS}

The first cool-down verified the thermal control system and tested the PMT array. For this the electrodes were replaced by a copper plate $8 \mathrm{~mm}$ 
above the PMT array. This plate had $31{ }^{241} \mathrm{Am}$ sources vacuum-sealed into it with a copper foil to stop $\alpha$-particles from reaching the xenon, leaving only low-energy (mainly $59.6 \mathrm{keV}$ ) photons. For subsequent tests the electrodes were re-installed.

\subsection{Cooling system, PMT array and scin- tillator output}

The initial cool-down, at $\sim 5^{\circ} \mathrm{C} /$ hour, uses 200 litres of liquid nitrogen $\left(\mathrm{LN}_{2}\right)$. Temperature is monitored at critical points. One on the cooling flange on the bottom of the target is the control temperature and is compared with a set temperature to automatically operate two valves: one exhausts straight from the gas volume of the $\mathrm{LN}_{2}$ reservoir, and one exhausts through the cooling ring. In normal operations the temperature is stable to better than $0.2^{\circ} \mathrm{C}$ and the $\mathrm{LN}_{2}$ usage drops to $\sim 20$ litres/day as expected.

Pulse height spectra, pulse waveforms and single photoelectron spectra (spes) were collected from all PMTs during the first cold-run both with the experiment DAQ electronics and with a pulse height analyser (PHA) set-up using a multichannel analyser (MCA). These confirmed operation of all 31 PMTs. Scintillation pulses from lowenergy ${ }^{241} \mathrm{Am} \gamma$-rays showed the characteristic decay time of $\sim 40 \mathrm{~ns}$. The spes show well resolved peaks and were used to set the amplifier gains to normalise all channels.

LXe scintillates in the vacuum ultraviolet (VUV), near $175 \mathrm{~nm}$. The VUV luminescence is produced by the decay of singlet and triplet states of the $\mathrm{Xe}_{2}^{*}$ excimer. These can be formed directly by excited atoms left by the interacting particle or as a result of recombination into an excited state along the particle track $[13,17]$.

Figure 3 shows MCA spectra taken from one PMT. The bottom spectrum was taken with the whole arrangement covered with liquid xenon. The two spectral features are the $59.6 \mathrm{keV}$ line from ${ }^{241} \mathrm{Am}$ and a blend of the $26.3 \mathrm{keV}{ }^{241} \mathrm{Am}$ $\gamma$-ray with the $30 \mathrm{keV}$ line from escape of Xe Kshell fluorescence photons. Using the MCA single photoelectron spectrum from this PMT gives a signal of $\sim 12 \mathrm{phe} / \mathrm{keV}$. There was no applied electric field. The top spectrum was taken with the liquid level between the source and the PMT window. The improved light collection (up to $\sim 17 \mathrm{phe} / \mathrm{keV}$ ) is a result of total internal reflection at the liquid gas interface. The two-phase resolution is $\sim 13 \%$ FWHM.

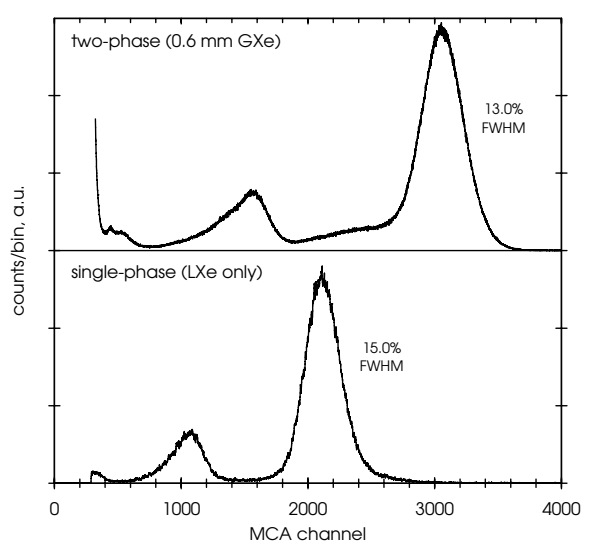

Figure 3. Typical MCA scintillation spectra with ${ }^{241} \mathrm{Am}$ sources a few $\mathrm{mm}$ above the PMTs. The two panels have different liquid xenon levels and the highest energy peaks in both are at $59.6 \mathrm{keV}$.

\subsection{Two-phase operation}

In two-phase operation a capacitive system probes the liquid xenon height with sub-mm accuracy at three locations. With the xenon filled to its nominal depth, but with no applied electric field, ${ }^{57} \mathrm{Co} \gamma$-ray spectra were recorded with an uncollimated source above the detector. A photoelectron yield of 5 phe $/ \mathrm{keV}$ was obtained from the fiducial volume, with a $\mathrm{FWHM} \simeq 25 \%$. This yield exceeds that predicted by Monte Carlo simulations (3.4-4.0 phe/keV) [2], which may indicate a higher LXe scintillation yield than previously (60 photons $/ \mathrm{keV}, W_{p h}=16.7 \mathrm{eV}$ ). After the zero field tests, $13.5 \mathrm{kV}$ was applied between cathode anode, giving a field of $3.0 \mathrm{kV} / \mathrm{cm}$ in the liquid. Figure 5 shows $\gamma$-ray interactions in the LXe. The fast scintillations are primary signals, S1. The second, broader signals, S2, occurs when the ionisation released at the inter- 
action site has drifted to surface and has been extracted into the gas. The time delay depends on the interaction depth and drift velocity at our fields $(\sim 2.5 \mathrm{~mm} / \mu \mathrm{s})$. The $\mathrm{S} 2$ width depends on the gas gap and electric field. Rise and fall times are a combination of extraction dynamics, diffusion and gas scintillation time-constant. S1 and
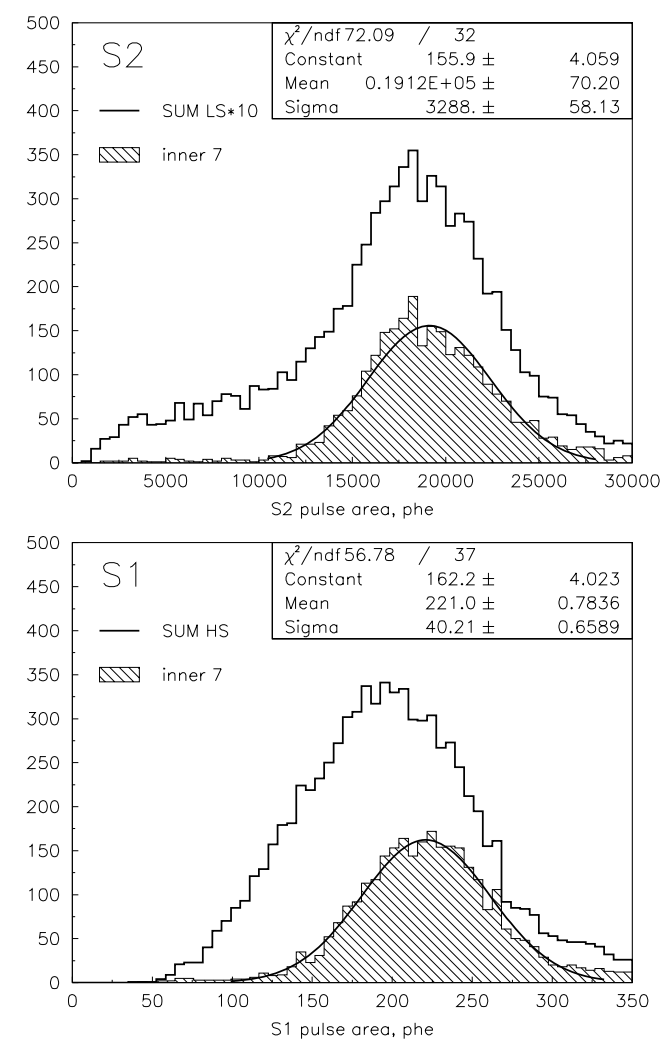

Figure 4. Spectra for S2 (top) and S1 (bottom) from an uncollimated ${ }^{57} \mathrm{Co}$ source above the instrument, with a field of $3.0 \mathrm{kV} / \mathrm{cm}$ in the liquid. The peak is a blend of the $122.1 \mathrm{keV}$ and $136.5 \mathrm{keV}$ lines $(85.6 \%$ and $10.7 \%$ respectively).

S2 ${ }^{57}$ Co spectra at $3.0 \mathrm{kV} / \mathrm{cm}$ are shown in figure 4, They use all PMTs, with individual gains and QEs equalised. The shaded spectra only include events with peak signals in one of the in- ner 7 PMTs. The 'collimated' S1 spectrum has a light yield of $1.8 \mathrm{phe} / \mathrm{keV} ; \sim 35 \%$ of the zero-field value. A single-Gaussian fit to the $122.1 \mathrm{keV}$ and $136.5 \mathrm{keV}$ blended lines gives $\mathrm{a} \simeq 40 \%$ FWHM.

A 3-D position recovery algorithm was developed from simulated datasets which provides sub$\mathrm{cm}$ resolution in the horizontal plane [18]. Even before this is applied to real data, the spatial sensitivity is well demonstrated in figure 5 , showing an event with two overlapping interactions. There are at least two primary and four secondary signals which, without position sensitivity can not be unambiguously separated. However, using individual PMT traces (left-hand in figure 5) it is obvious that two double-Comton scatters have happened in very different parts of the detector.
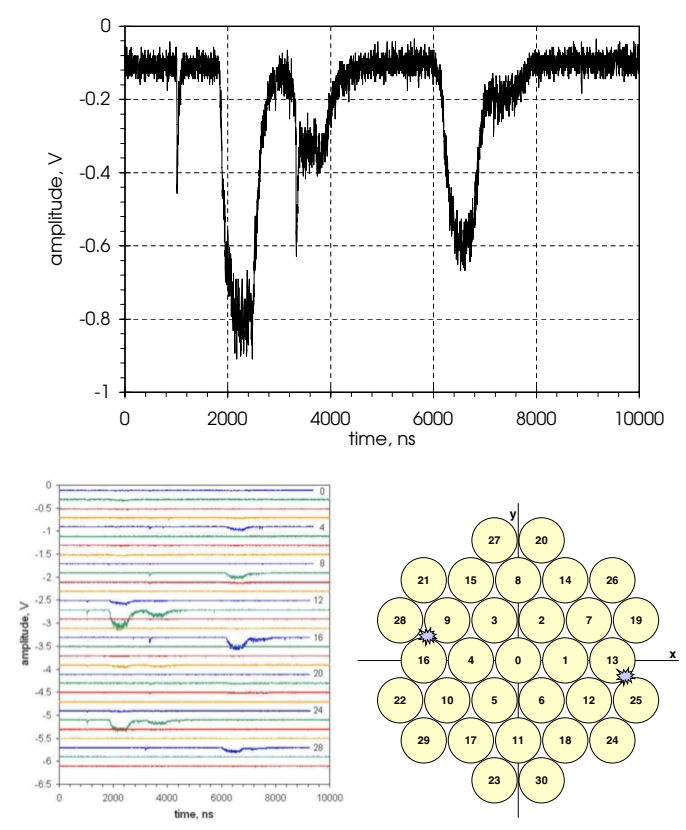

Figure 5. Top: Summed waveforms from two overlapping $\gamma$-ray events. Lower: Individual waveforms from the 31 PMTs and reconstructed positions. The symbol size corresponds to the position resolution.

Demonstration of discrimination is given in figure 6 , showing the $\mathrm{S} 2 / \mathrm{S} 1$ ratio when a $10 \mathrm{mCi}$ 
Am-Be $(\alpha, n)$ source is placed above the detector. The field within the liquid was $3 \mathrm{kV} / \mathrm{cm}$ as before. Discrimination between elastic recoils (population A) and $\gamma$-ray interactions (C) is clearly demonstrated. Population B is from neutron inelastic scattering off ${ }^{129} \mathrm{Xe}$ nuclei; some (quenched) nuclear recoil energy is augmented by a $40 \mathrm{keV} \gamma$-ray from nuclear de-excitation.

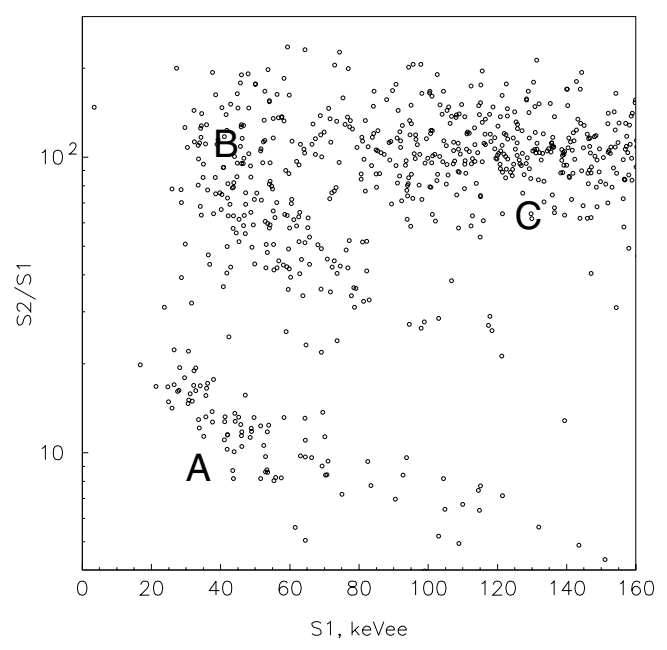

Figure 6. S2/S1 ratio as a function of S1 for singlescatter events with an Am-Be source. For elastic nuclear recoil events (population A) the energy scale must be multiplied by 1.95 , coming from a combination of the inverse quenching factor, $(1 / 0.19$ [14-16]) and the relative S1 suppression factor at our operating field $(\sim 0.37)$. Population $\mathrm{C}$ are $\gamma$-rays.

\section{ACKNOWLEDGEMENTS}

This work has been funded by the UK Particle Physics And Astronomy Research Council (PPARC). We would like to acknowledge the superb copper machining achieved within the Imperial College Physics Department workshop led by R. Swain, and the development of new welding techniques by The Welding Institute.

\section{REFERENCES}

1. D. Yu Akimov et al., Astropart. Phys., in press (2006)

2. H. M. Araújo et al., Astropart. Phys., in press (2006) - (arXiv:astro-ph/0603243)

3. G. J. Alner et al., New Astronomy Reviews 49 (2005) 259.

4. G. J. Alner et al., Nucl. Instrum. Meth. A 555 (2005) 173

5. B. A. Dolgoshein, V. N. Lebedenko \& B. U. Rodionov, JETP Lett. 11 (1970) 513

6. T. J. Sumner et al., Proc. 26th Int. Cosmic Ray Conf., D. Kieda, M. Salamon \& B. Dingus (Eds), 2 (1999) 516.

7. A. S. Howard et al., Proc. 3rd Int. Workshop on the Identification of Dark Matter, N. J. C. Spooner \& V. Kudryavtsev (Eds), Singapore: World Scientic (2001) 457.

8. D. Yu Akimov et al., Proc. 4th Int. Workshop on the Identification of Dark Matter, N. J. C. Spooner \& V. Kudryavtsev (Eds), Singapore: World Scientific, (2003) 371.

9. T. J. Sumner, New Astronomy Reviews 49 (2005) 277.

10. D. Davidge, PhD Thesis, University of London (2003).

11. J. Dawson PhD Thesis, University of London (2003).

12. E. Aprile et al., (2006) - (ArXiv: astro$\mathrm{ph} / 0601552$ )

13. S. Kubota et al., Phys. Rev. B 20(8) (1979) 3486.

14. D. Akimov et al., Phys. Lett. B 524 (2002) 245

15. E. Aprile et al., Phys. Rev. D 72 (2005) 072006

16. V. Chepel et al., ArXiv:physics/0512136, (2005)

17. A. Hitachi et al., Phys. Rev. B 27(9) (1979) 5279 .

18. A. Lindote et al., Nucl. Instrum. Meth. A in press (2006) 